\title{
Energetic neutral atoms from the Sun: an alternative interpretation of a unique event ${ }^{\star}$
}

\begin{abstract}
G. M. Simnett
School of Physics and Astronomy, University of Birmingham, B15 2TT, UK

e-mail: gms@star.sr.bham.ac.uk

Received 2 January 2011 / Accepted 9 May 2011

ABSTRACT

The high temperature of the solar corona results in virtually complete ionization of the light elements and a high degree of ionization of the heavier elements. Therefore it is not expected that many neutral atoms should be emitted from the Sun, and certainly not with high kinetic energy. A particle event associated with the first major flare of the current solar cycle, on 5 December 2006, has been interpreted as containing energetic neutral hydrogen atoms (ENA) of at least a few MeV. The ENAs were identified as such on account of their arrival direction at $1 \mathrm{AU}$, which was from the solar direction; the lack of atoms heavier than hydrogen; and the timing of their arrival, which was consistent with emission at the time of the flare X-ray burst. The observations were made from the two STEREO spacecraft which were near the Earth at the time. However, the EPAM instrument on the ACE spacecraft, which is in orbit around the $L_{1}$ Lagrangian point some $1.5 \times 10^{6} \mathrm{~km}$ away from the Earth towards the Sun, observed a pulse, or precursor, of electrons of energies of at least $50 \mathrm{keV}$ but approximately one hour earlier than the pulse of ENAs at STEREO. Later ACE and STEREO detected detected a major charged particle event which is presumably associated with the 5 December flare. The relative times of the onsets of the energetic particles in both the precursor and the main solar energetic particle event at the STEREO spacecraft and ACE were consistent with corotation of the interplanetary magnetic field if the particles were the same population propagating, and probably trapped, within the field. The precursor proton intensity detected by STEREO was below the threshold of the ACE/EPAM detectors. We conclude that the interpretation of the particles seen by STEREO as energetic neutral atoms is suspect.
\end{abstract}

Key words. Sun: particle emission - Sun: flares

\section{Introduction}

In a recent paper by Mewaldt et al. (2009) it was argued that a burst of protons seen $\sim 1 \mathrm{~h}$ following the onset of a GOES-class X9 flare on 5 December, 2006, at E79S06 degrees on the visible solar disc, had travelled from the Sun as neutral hydrogen atoms before entering their detector and becoming ionized. They suggested that energetic neutral atoms (ENA) were produced from energetic flare-accelerated protons which had undergone charge exchange with heavy ions present in the corona or by radiative recombination with ambient coronal electrons. In support of this interpretation was the fact that the protons they detected were not accompanied by heavier ions, such as He nuclei. Their observations were made from particle detectors on the twin STEREO spacecraft, which were still close to the Earth on 5 December, 2006. This intriguing interpretation may possibly be true, but we believe it unlikely for reasons we shall discuss.

The Advanced Composition Explorer (ACE) spacecraft is sunward of the Earth, in orbit around the $L_{1}$ Lagrangian point. Around an hour before the supposed ENA detection at STEREO the EPAM instrument on ACE detected a burst of nearrelativistic electrons, which lasted around $15 \mathrm{~min}$ at an intensity above the ambient background. This burst we refer to as the precursor (following Mewaldt et al. 2009).

The electron pitch angle distribution was highly unusual, which first made us suspect that the data might not be accurate.

* Appendix is available in electronic form at http://www . aanda . org
However, the response to the subsequent event was normal, so we may therefore accept the precursor data at its face value. The detected electrons had a pitch angle distribution showing that they were predominantly perpendicular to the local magnetic field, which would be consistent with a trapped distribution. How this might arise is difficult to say, and further discussion is beyond the scope of the present paper. They were seen to start arriving around $3 \mathrm{~h}$ and $23 \mathrm{~min}$ before the onset of the energetic particles from the X9 flare.

The precursor seen by STEREO started $3 \mathrm{~h}$ and $15 \mathrm{~min}$ before the onset of the flare particles. It would be too much of a coincidence for the two events at ACE to be separated by essentially the same time as the two events seen by STEREO if they were independent. Therefore it is worth examining the event in more detail, especially for an alternative explanation that fits all the data.

Particles from poorly-connected flares may propagate onto interplanetary magnetic field lines which do not intersect the Earth. As the Sun rotates, magnetic structures populated with energetic flare particles may corotate past the Earth. This results in the onset at the Earth of particles accelerated in flares close to the solar east limb being typically delayed by some hours from the onset of the flare. We believe that the main particle increase in the 5 December event is corotating and we shall discuss this in more detail below. A signature of a corotating event is the simultaneous increase at an observing spacecraft of particles of different velocity. In other words, the fine structure in the intensity-time profiles appears simultaneously at all energies. 
There is frequently backscatter, generally from a radial distance beyond the Earth, which can result in effective trapping of the particles within the interplanetary magnetic field.

There are two criteria which are highly relevant to the interpretation of unusual events: composition, including electrons; and the timing of the arrival at the observing spacecraft of the particles. Neutral particles coming from the Sun are not affected by the interplanetary magnetic field and should be detected at times commensurate with their speed. A reasonable hypothesis is that energetic particles are emitted from the Sun around the time of the maximum of the flare soft X-ray emission, say \pm 10 min.

The event we are discussing has three possible interpretations. (1) it is caused by ENAs; (2) it is caused by neutrons; (3) it is an unusual minor particle event caused by trapped particles propagating in some kind of plasmoid which is devoid of heavier energetic ions at energies that could be detected. In the latter case it is feasible that the particles were in a corotating magnetic structure which had been populated with energetic particles by some prior (10s of minutes) solar transient event, which may easily be closely related to a solar disturbance which is part of the precursor to the major flare. We present some data from the onset of the subsequent major solar particle event to demonstrate that the EPAM instrument is functioning properly. The lack of contamination by solar X-rays in the prime EPAM electron data is shown in the Appendix.

\section{Energetic particle observations at the ACE spacecraft}

The charged particle observations we discuss were made by the Electron, Proton and Alpha Monitor (EPAM) instrument on ACE, which has a suite of five detectors responding to electrons from $\sim 40-300 \mathrm{keV}$, ions from $\sim 50-4800 \mathrm{keV}$ and nuclei above $\sim 0.5 \mathrm{MeV} /$ nucleon. For a description of the EPAM instrument and the nomenclature used to identify the data see Gold et al. (1998). The instrument was built in the early $1980 \mathrm{~s}$ as the spare unit for the HI-SCALE instrument flown on Ulysses (Lanzerotti et al. 1992) and knowledge of the precise energies for the various channels is the best available, but may be somewhat inaccurate.

The ACE spacecraft is orbiting the $L_{1}$ point, around $1.5 \times$ $10^{6} \mathrm{~km}$ towards the Sun, while on 5 December, 2006 the STEREO spacecraft were still both close to the Earth. Thus for a nominal Parker spiral magnetic field, aligned at $45^{\circ}$ to the Earth-Sun line at $\sim 1 \mathrm{AU}$, a corotating structure would pass ACE around $60 \mathrm{~min}$ before it passed the Earth. The STEREO spacecraft were in transfer orbits somewhere between the Earth and the Moon, but uncertainty in their precise position would not affect this conclusion significantly, nor would minor departures of the magnetic field from the nominal Parker spiral.

The relative positions of the ACE and STEREO spacecraft provide a strong constraint on the interpretation of the precursor event. Neutral particles or photons travelling from the Sun will do so radially. Therefore a burst of solar photons will reach the Earth $\sim 5 \mathrm{~s}$ later than at ACE; similarly, a burst of neutral particles travelling at, say $0.1 c$, will reach the Earth about $50 \mathrm{~s}$ after reaching ACE. With these constraints in mind, we shall now review the charged particle data from ACE and compare it with that published by Mewaldt et al. (2009) from STEREO.

On 5 December 2006 the Sun produced a major flare from an active region at E79S06 degrees on the visible solar disc. All times in this paper are on 5 December, 2006 unless otherwise stated. The flare was classified as X9 by the GOES soft X-ray monitor and was one of the largest events from solar cycle 23. The soft X-ray maximum was around 10:30 UT (Solar Geophysical Data, US Dept. Commerce, Boulder, CO). Energetic electrons were detected at ACE as the onset of a major event at around 13:50 UT. However, at 10:28 UT a small precursor event was detected. At STEREO energetic protons from the flare were detected around 14:45 UT and a precursor was detected around 11:30 UT. It is the latter increase that Mewaldt et al. (2009) have interpreted as ENA. As this was early in the STEREO mission electron observations from STEREO were unfortunately not available.

ACE is spinning at $5 \mathrm{rpm}$ and the particle intensities are monitored in four sectors for some channels and eight sectors for others (Gold et al. 1998). Figure 1 shows the onsets of both the precursor and the main event at ACE, as measured by the deflected electron channels on EPAM at 38-53 keV (DE1), 53-103 keV (DE2) and 53-62 keV (E1'). The four sectors for the electrons are plotted and the precursor is clearly anisotropic. The arrows in the top panel of Fig. 1 are drawn at 10:27 and 13:50 UT. It is clear that electrons from the precursor event are not detected by EPAM above $53 \mathrm{keV}$ in the roughly sunward facing detectors. In the LEFS60 detector (Fig. 1c) the presursor is detected mainly in sectors 4 and 5. The energetic particle intensities at ACE had been around background levels for over 2 days before the precursor increase discussed here, so that it is unlikely that the precursor is associated with anything other than the major X9-class flare referred to above.

The axis of the LEFS150 sensor is pointing $\sim 30^{\circ}$ away from the direction of the Earth. However, the precursor is seen in the 58-104 keV electron channel of LEFS150 detector (E2), shown in Fig. 1d. It is visible in only two of the four sectors, but the increase is over two orders of magnitude above the background. The LEFS150 detector has a high noise level, which exceeds the threshold of the lowest energy channel. A simplistic, ad hoc, interpretation of the data would be to subtract a noise level of $\sim 30 \mathrm{keV}$ from the energy channel boundaries, which would then put the energy boundaries for the E2 channel down from $58-104 \mathrm{keV}$ to $28-74 \mathrm{keV}$, which would be consistent with the data from the other electron detectors.

The increase starting around 13:50 UT is interpreted as particles associated with the X9 flare. The intensity fluctuations of these flare particles are seen simultaneously in all energy channels, showing that the increases are spatial, rather than temporal, as there is no velocity dispersion. This is the signature of a corotating event. Note the slight rise from the background level for about an hour before the main increase.

We have put a black bar in Fig. 1a between the arrows corresponding to the onsets of the precursor and the main event. In Fig. 1b we have marked with arrows the onset time of the precursor and the onset of the main particle event at STEREO. We have copied the black bar onto Fig. 1b. Within the accuracy of the timing it is clear that the separation between the two sets of arrows is the same. The onsets of the main event, which is certainly corotating, are in no doubt, and these times serve to establish the corotation delay between ACE and STEREO.

Figure 2 shows an overview of the event as seen in the spin averaged intensity for all four of the deflected electron channels (see figure caption). There is no significant velocity dispersion visible in these data after 13:50 UT, which is when the intensities rise sharply. However, the electron intensities undergo a small increase after around 12:00 UT which does have velocity dispersion, just visible in Fig. 2. The dashed line is drawn by eye to aid in the identification of this phenomenon. We interpret this as evidence that there is some continuing emission of electrons 
G. M. Simnett: Solar energetic neutral atoms
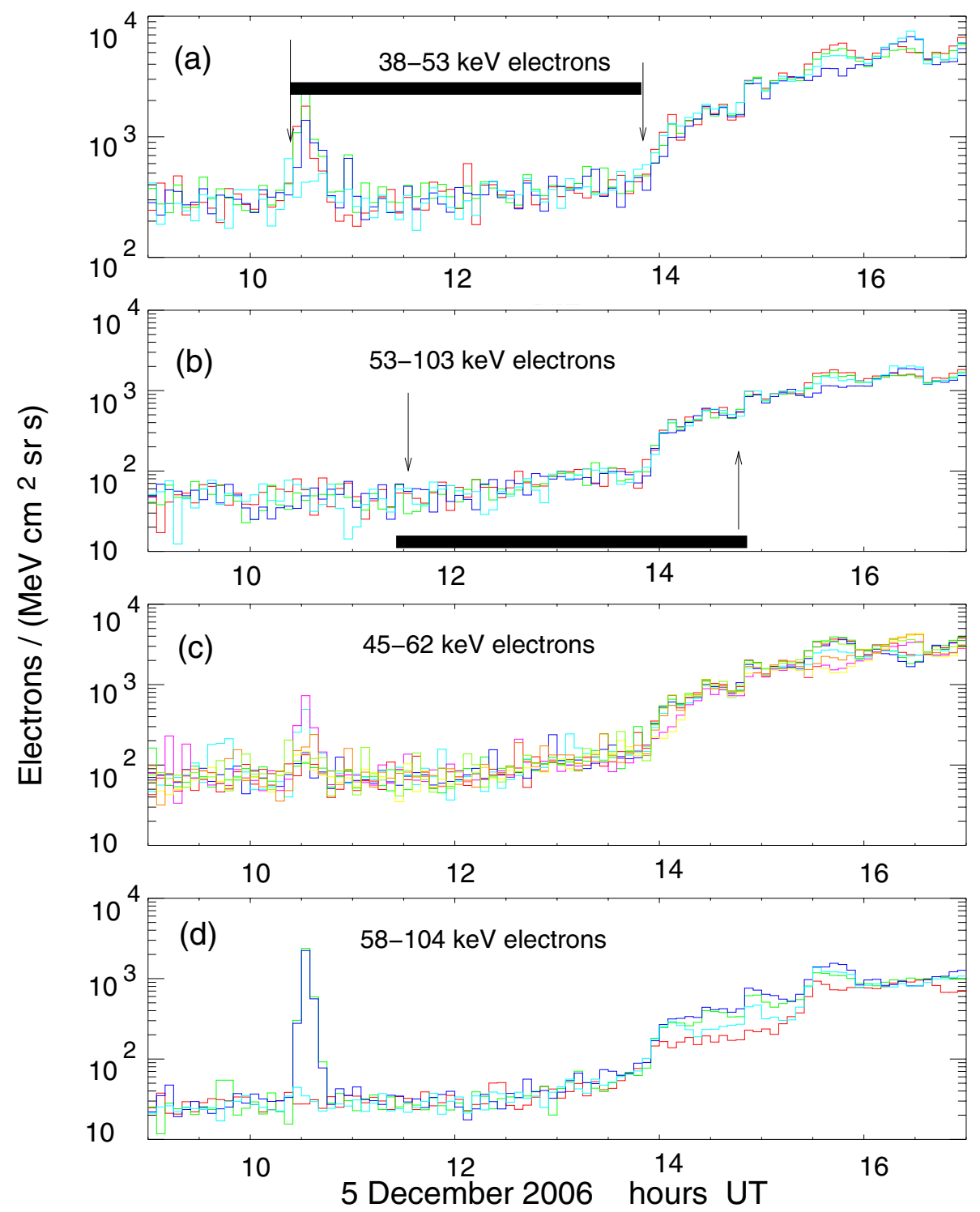

Fig. 1. The onset of the precursor and the main event at ACE. The upper trace a) shows the four sectors of the deflected electrons (DE1); and b) shows the same, but for channel DE2. The arrows in a) are drawn at 10:27 UT on 5 December, 2006, and 13:50 UT. Trace c) shows the eight sectors of the LEFS60 channel E1'; and d) shows the LEFS150 E2 channel (see text for discussion of energy channel boundaries). The arrows in $\mathbf{b}$ ) are drawn at the onset times of the precursor (11:30 UT) and the main event (14:45 UT) at STEREO (Mewaldt et al. 2009). The dark bar in a) covers the time between the precursor and the main event onsets and is exactly reproduced in $\mathbf{b}$ ). The data are plotted as 5 min averages and the nominal energy band is indicated in each plot (see text). from the Sun at a low level and from parts of the corona adjacent to that from which most of the particles were emitted (the main event) although other interpretations may be equally true. Note the good correlation of the intensity fluctuations in all four electron channels during the main event. This shows that the spacecraft is sampling primarily a spatial variation of the electron intensity, rather than a time-dependent increase. This is consistent with the data shown in Fig. 1 for the four sectors. These data also show that EPAM is functioning correctly, apart from the possible uncertainty in the numerical boundaries of the energy channels.

The magnetic field is shown in the lower panels of Fig. 2 for the period covering this event. We are using spacecraft coordinates, where the reference direction is the Sun-spacecraft $\left(0^{\circ}\right.$ azimuth $)$ and the reference plane is the ecliptic. We note that the field azimuth direction is relatively constant for the period $10-16 \mathrm{~h}$ UT on 5 December. There is nothing to suggest that the field should be capable of trapping or modifying the unusual particle intensities we are discussing. The solar wind speed was around $310 \mathrm{~km} \mathrm{~s}^{-1}$.

In Fig. 3 we plot the pitch angle distribution for the LEFS60 E1' channel, covering electrons from $45-62 \mathrm{keV}$. The abscissa is the cosine of the angle the axis of the detector makes with the magnetic field at the centre of the data accumulation time. The ordinate is the particle intensity normalised to 1 at the peak intensity. The actual intensity is $\mathrm{S}$, which is given at the top of each panel. EPAM doesn't have coverage in the backward (Earth-pointing) hemisphere in the lowest channel covering 44-58 keV electrons on account of noise in the LEFS150 detector. Figure 4 shows the pitch angle distribution of the LEMS150 E2 electron channel, which nominally covers the energy range 58-104 keV, from 10:20-10:44 UT. The data for the electron pulse indicate a very anisotropic distribution, with a minimum along the magnetic field direction. Note that the background pitch angle distribution shown in the first panel from 10:20-10:26 UT is field-aligned.

For the precursor event the electron distribution is maximum away from the magnetic field vector, which is suggestive of a trapped distribution. This is rarely seen in the EPAM data.

For comparison, in Fig. 5 we give the electron pitch angle distribution at various times near the onset of the main event. The data are plotted for successive periods of $15 \mathrm{~min}$ from 13:50-15:10 UT. The data show that the electron intensity is somewhat anisotropic, with a minimum along the magnetic field direction, which is approximately along the nominal Parker 

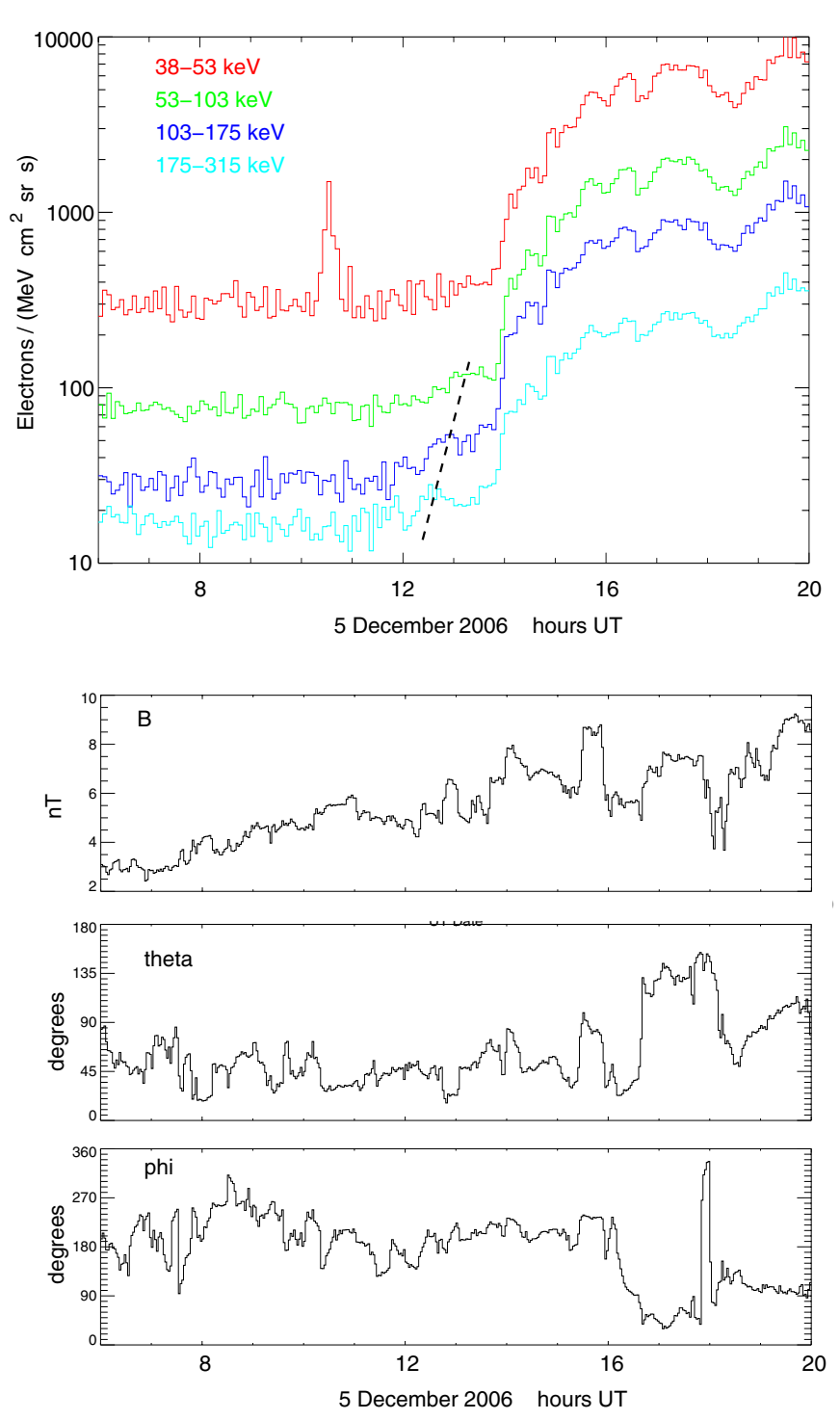

Fig. 2. Upper panel: the intensity-time history of the precursor event and the main event at ACE, seen in the four deflected electron channels on EPAM. The energies of the channels are 38-53 keV (red), 53-103 keV (green), 103-175 keV (blue) and 175-315 keV (cyan) and they are plotted as $5 \mathrm{~min}$ averages and spin averages. The dashed line indicates the possible velocity dispersion seen before the main onset at around 13:50 UT. Lower panels: the magnetic field at ACE, plotted as the magnitude (B), colatitude (theta) and azimuth (phi).

spiral towards the Sun. These data show that the EPAM instrument is measuring the onset of the energetic electrons from the major east limb flare in a manner which is consistent with previous major flare-associated events.

The precursor event is quite remarkable in that it has several features which are very difficult to interpret. The EPAM instrument has some detectors which have a slightly ambiguous response to electrons and ions (see Gold et al. 1998; Lanzerotti et al. 1992). One might question whether the unusual behaviour in the EPAM electron data could be an artefact, caused, for example, by energetic solar photons. While this occurs in the LEMS30 detector, it has never been known to occur in the deflected electron channels. In the Appendix we show data from four large GOES X-class flares which occurred at various times throughout the ACE mision, plus the X6.5 flare that occurred on 6 December, 2006. Thus we are confident that the
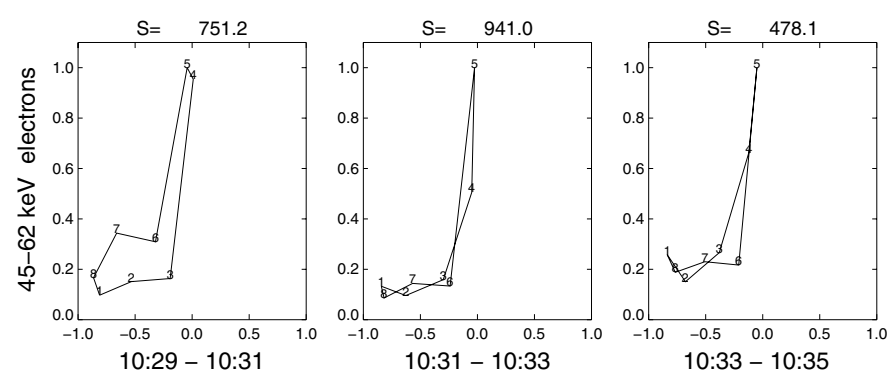

Fig. 3. The electron pitch angle distribution from 10:29-10:35 UT on 5 December in the LEMS60 E1' channel.
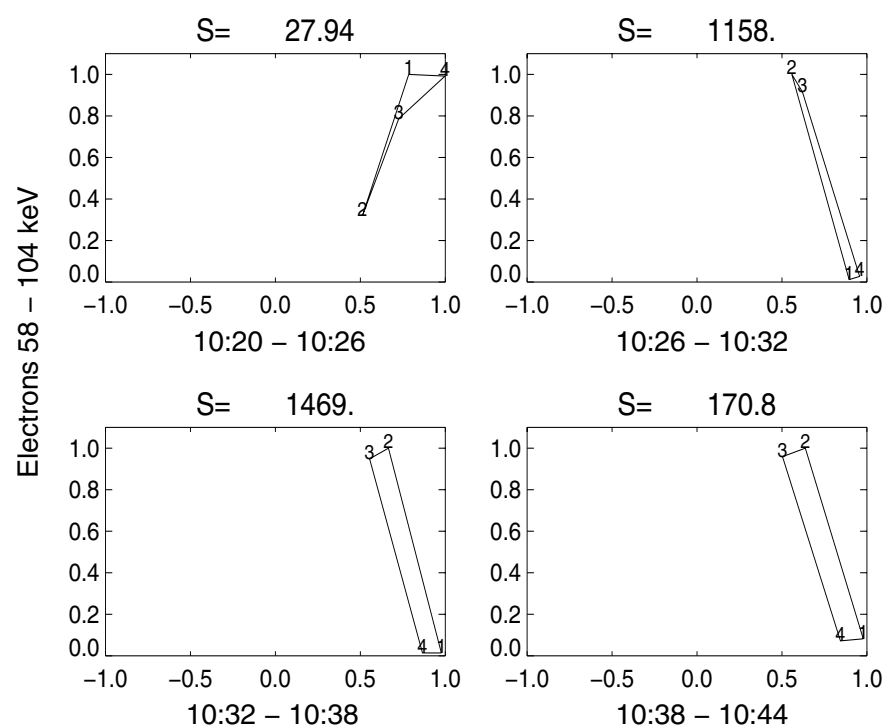

Fig. 4. The 58-104 keV electron pitch angle distribution at various times during the precursor (see text for discussion of the energy channel boundaries). Notice that the electrons have a strong minimum along the (+) magnetic field direction. The data are plotted as 6 min averages of the E2 channel.

magnetically-deflected electrons do not suffer from any contamination and thus the intensity and pitch angle distributions truly represent those of electrons. Furthermore, the LEFS60 and LEFS150 detectors, which detect electrons, are generally reliable and uncontaminated, especially for the channels in the middle of the response function.

The magnetosphere might conceivably be the source of the electrons seen by ACE. However, if this were the case, then we would expect the electrons to be field-aligned and coming from the anti-solar direction. Figure 4 shows that they are not fieldaligned. Also, a direct magnetic connection between ACE and STEREO would be an extreme departure from a nominal Parker field.

\section{Discussion}

The similarity between the intensity-time profile of the precursor event seen at ACE with that experienced by STEREO (Mewaldt et al. 2009, their Fig. 1) establishes beyond reasonable doubt that the spacecraft are experiencing the same event, separated in time simply because they are well-separated in space. The presence of electrons is difficult to understand if the precursor event is caused by energetic neutral hydrogen atoms. One might postulate, however, as did Mewaldt et al., that the detected particles, 

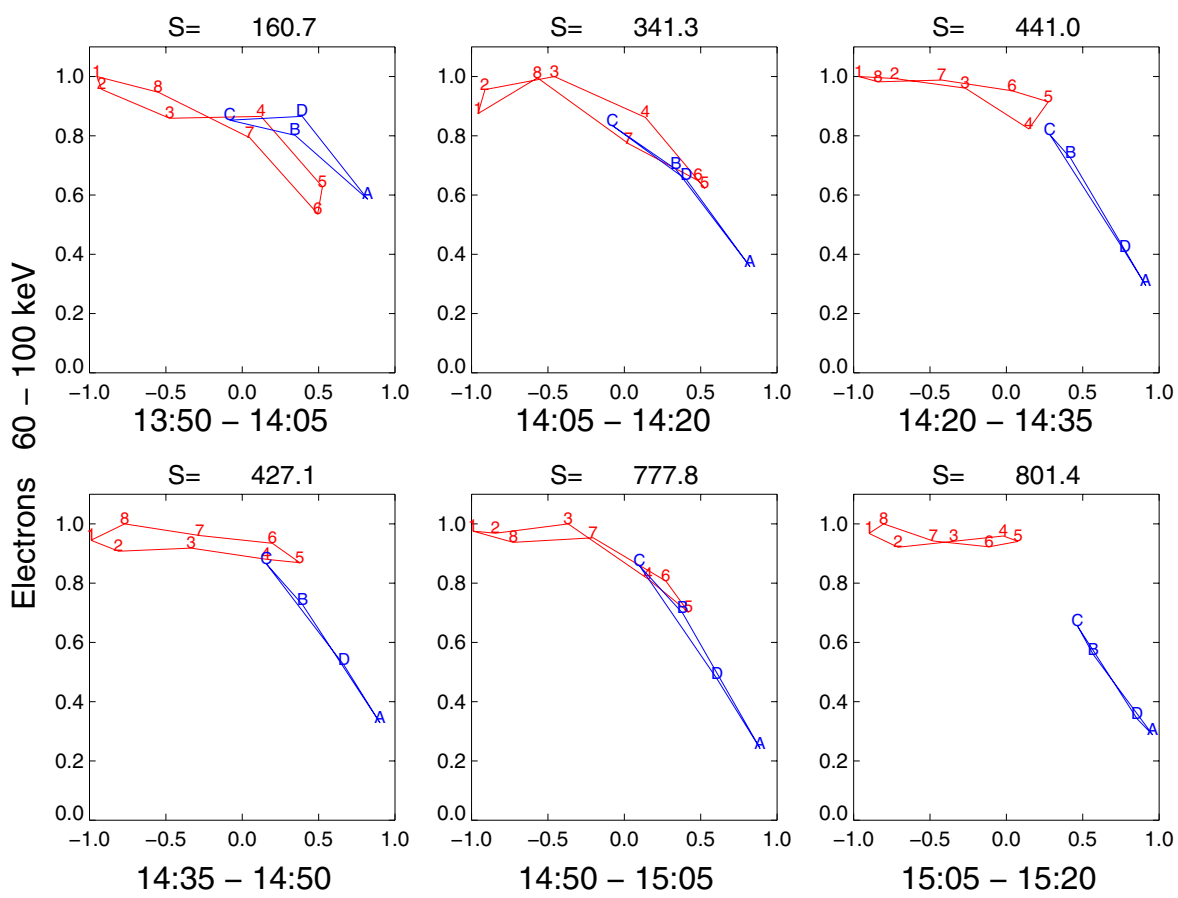

Fig. 5. The $\sim 60-100 \mathrm{keV}$ electron pitch angle distribution at various times around the onset of the main event. Notice that the electrons have a weak anisotropy, with a minimum along the $(+)$ magnetic field direction. The data are plotted as 15 min averages of the E2' and E2 channels. The DE2 channel has similar pitch angle coverage to the E2' channel and for simplicity is not shown.

be they protons or electrons, are the result of neutron decay. This phenomenon has previously been discussed at length by Dröge et al. (1996). They show that the electrons from energetic neutron decay have an energy spectrum which peaks around $300 \mathrm{keV}$ (see Dröge et al. 1996, their Fig. 2). This is inconsistent with the spectrum of the detected electrons at ACE, which do not exceed $100 \mathrm{keV}$ at the sensitivity level of the detector and therefore must have an energy spectrum that is steeply falling above, say, $50 \mathrm{keV}$. Mewaldt et al., for different reasons, also rejected the neutron decay hypothesis.

We have shown above that the precursor detected by EPAM is a very unusual event, just as that detected by STEREO is also unusual. At STEREO the main particle increase, presumably from the X9 flare at E79S06 on the visible solar disc, started around 14:45 UT (Mewaldt et al. 2009). The precursor, which Mewaldt et al. attribute to neutral atoms, started around 11:30 UT. The timing of the events at the various spacecraft supports our hypothesis that they are the same. The corotation delay between ACE and Earth is about $1 \mathrm{~h}$, with ACE leading. This is consistent with the ACE-STEREO delay, both for the precursor and the main event.

Finally, we might suppose that any neutral atoms detected by STEREO could also be detected by EPAM. When would this be? For illustrative purposes we will perform the calculation for atoms of kinetic energy $4.7 \mathrm{MeV}$, which have a velocity of $0.1 \mathrm{c}$. ACE is $\sim 1.5 \times 10^{6} \mathrm{~km}$ closer to the Sun, and thus should encounter the hypothetical neutral atoms $1.5 \times 10^{6} /\left(0.13 \times 10^{5}\right) \mathrm{s}$, or $50 \mathrm{~s}$, before STEREO. Throughout the period between the end of the precursor and the onset of the main particle event the ions at EPAM were at background levels. However, the sensitivity of EPAM is at the level of $\sim 5 \times 10^{-2}$ particles/( $\left.M e V \mathrm{~cm}^{2} \mathrm{~s} \mathrm{sr}\right)$ at around $1 \mathrm{MeV}$, so it might not be sensitive enough to detect the particles reported by Mewaldt et al. (2009).

We are now faced with the conclusion that the precursor at EPAM is neither from neutral atoms as it includes nearrelativistic electrons, nor from neutrons, as the electron spectrum is contrary to that expected from neutron decay. Therefore we are left with the third of our options given in the introduction. The precursor is very unusual in that the particle distributions seen by
EPAM resemble narrow beams of particles, which are detected in some sectors but not others, and have a sharp upper energy cut-off for electrons. There is no evidence for the precursor in DE2. In the LEFS150 detector the precursor is clearly seen in E2 (Fig. 2), which nominally responds to $58-104 \mathrm{keV}$. However, we speculate that due to the increased noise level in this detector, which makes the lowest channel unusable, the channel energy boundaries may be lower than nominal.

By the time the corotating structure reaches STEREO, the protons trapped within it are predominantly coming from the sunward direction, which Mewaldt et al. (2009) find extremely difficult to explain other than by neutral particles. Unusual as this may seem, we can only point out that the event detected by EPAM is also difficult to explain. But a provenance in neutrons or neutral atoms is ruled out. We should emphasise that the magnetic field, as shown in Fig. 2, does not suggest that there could be any significant local particle acceleration taking place.

The composition of the precursor is unusual, in that Mewaldt et al. (2009) do not detect any energetic ions heavier than protons. EPAM can also measure the composition of energetic particles. Near the onset of the main event, around 15:00 UT the ratio of $0.52-1.73 \mathrm{MeV}$ protons to $0.39-6.98 \mathrm{MeV}$ helium nuclei measured by EPAM was around 6000. (NB This number is uncorrected for the instrument response.) Near the maximum of the particle event on 8 December this ratio had dropped to $\sim 90$. Thus near the onset of the main event the energetic nuclei are deficient in helium, which is also the case at STEREO.

\section{Conclusions}

The precursor energetic particle increase seen on 5 December 2006 is a very interesting and unusual event. The data we have analysed from ACE/EPAM are unique in our experience, and one might be tempted to reach an intriguing and plausible explanation, as was done by Mewaldt et al. (2009). We have considered three possible scenarios to account for it, namely ENAs, solar neutrons produced at the Sun following an X9 flare, and an unusual particle event propagating though the interplanetary medium from the Sun to beyond the Earth. The neutral 
atom hypothesis can be eliminated as it is accompanied by nearrelativistic electrons and if it were to relate to the ENAs suggested by Mewaldt et al., it comes about an hour too early. Also, the neutron hypothesis can be eliminated as neutron decay would be accompanied by electrons of much higher energy than seen by EPAM.

The major energetic particle event, which had an onset at ACE at around 13:50 UT, has an intensity-time profile similar to that seen by STEREO to start at 14:45 UT. The fluctuations in particle intensity for different energy (velocity) particles are observed simultaneously (see Figs. 1 and 2, and Mewaldt et al. 2009, their Fig. 1), proving that the spacecraft are sampling spatial, rather than temporal, effects. Therefore the main particle event is corotating with the solar rotation speed, through the interplanetary medium. We have shown that the nominal corotation delay between ACE and the Earth is around one hour, with ACE leading. This nominal time difference is almost exactly what is seen for both the precursor and the main event.

The most plausible explanation for the precursor is that it contains energetic protons (detected by STEREO) and near relativistic electrons (detected by ACE) emitted from the Sun before, but close to the time of the X9 flare. It is possible that these particles are accelerated in the interplanetary medium within an unusual magnetic structure, but we shall not discuss this speculation further. The magnetic structure which is corotating with the Sun appears to contain trapped electrons; any electrons with small pitch angles have been lost. The proton intensity, if present, appears to be below the threshold of the EPAM detectors.

If the rather unusual electron event detected by ACE/EPAM is entirely separate from the proton event detected by STEREO, then we need two, previously unknown, interpretations of these data. If they are linked by an unusual corotating interplanetary event then only one new interpretation is needed (Occam's razor). The lack of heavy nuclei in the STEREO data suggests that some type of resonant acceleration process may be needed, although we would have no objection to other mechanisms. We conclude that the interpretation of Mewaldt et al. (2009) that they have detected energetic neutral hydrogen atoms from the Sun, is suspect, as we would then have to find separate solution to the electron event at EPAM.

Acknowledgements. The author wishes to thank the referee for helpful comments.

\section{References}

Dröge, W., Ruffolo, D., \& Klecker, B. 1996, ApJ, 464, L87

Gold, R. E., Krimigis, S. M., Hawkins, S. E., III, et al. 1998, Space Sci. Rev., 86, 541

Lanzerotti, L. J., Gold, R. E., Anderson, K. A., et al. 1992, A\&A, 92, 349

Mewaldt, R. A., Leske, R. A., Stone, E. C., et al. 2009, ApJ, 693, L11

Simnett, G. M. 2005, J. Geophys. Res., 110, A09S01

Pages 7 to 8 are available in the electronic edition of the journal at http://wWw. aanda.org 


\section{Appendix A: Response of EPAM to solar X-rays}

It is well known that energetic particle instruments using solid state detectors experience a response to solar X-rays if they are pointed at the Sun. Therefore it is customary to ignore the data from such detectors at times of intense solar X-ray activity. The LEMS30 detector on the EPAM instrument has two sectors out of four which scan the Sun as the spacecraft spins. The magnetically-deflected electrons enter the instrument through the same aperture as the ions detected by LEMS30, but a magnet sweeps them into a well-shielded detector which has negligible response to solar X-rays. In this appendix we demonstrate the integrity of the deflected electron data by showing the responses of both the electron detector and the LEMS30 ion detector at times of intense solar X-ray activity.

Table A.1. Large X-ray flares which contaminate sunward-facing detectors.

\begin{tabular}{lcccc}
\hline \hline Date & $\begin{array}{c}\text { X-ray flare } \\
\text { GOES class }\end{array}$ & $\begin{array}{c}\text { Time of } \\
\text { size }\end{array}$ & $\begin{array}{c}\mathrm{H} \alpha \\
\text { maximum }\end{array}$ & $\begin{array}{c}\text { Flare } \\
\text { location }\end{array}$ \\
\hline 4 November 1997 & X2.1 & $05: 58$ & 2B & S13W33 \\
2 April 2001 & X20 & $21: 49$ & & N17W78 \\
15 April 2001 & X14.4 & $13: 50$ & 2B & S20W85 \\
28 October 2003 & X17.2 & $11: 10$ & 4B & S16E08 \\
6 December 2006 & X6.5 & $18: 47$ & 3B & S06E63 \\
\hline
\end{tabular}

The flares we have chosen are given in Table A.1. They are all GOES X-ray class X-flares, and three of them are among the largest of the solar cycle 23. Figure A.1 shows the intensitytime history from 05:45-06:45 UT on 4 November 1997 for EPAM channnels P4 and DE1, which are designed to measure $193-315 \mathrm{keV}$ ions and $38-53 \mathrm{keV}$ electrons. This flare was GOES class X2.1 and it was preceded by a relatively quiet period, with a low ambient background of charged particles. The data are plotted showing the four sectors independently. The detected ions and electrons enter the instrument though the same aperture, which for two of the sectors (blue and green) sweep past the Sun. It is clear that the P4 channel is responding to solar X-rays in these two sectors, but not in the other two sectors,

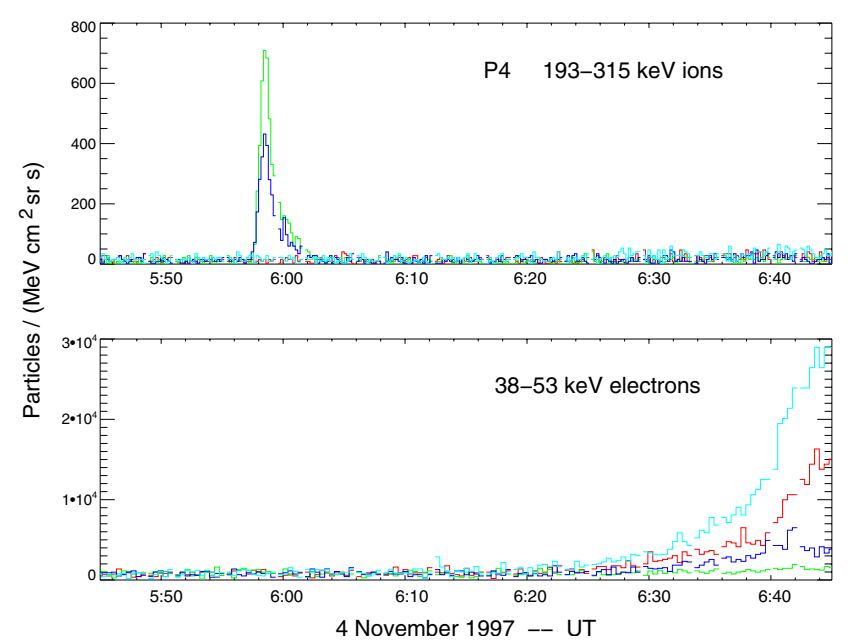

Fig. A.1. Contamination in the LEMS30 detector from solar X-rays on 4 November 1997. The upper panel is the $\mathrm{P} 4$ channel, which is designed to respond to ions with energies between 193 and $315 \mathrm{keV}$. The lower panel are the magnetically-deflected electrons, which enter the EPAM instrument through the same aperture as the LEMS30 ions.

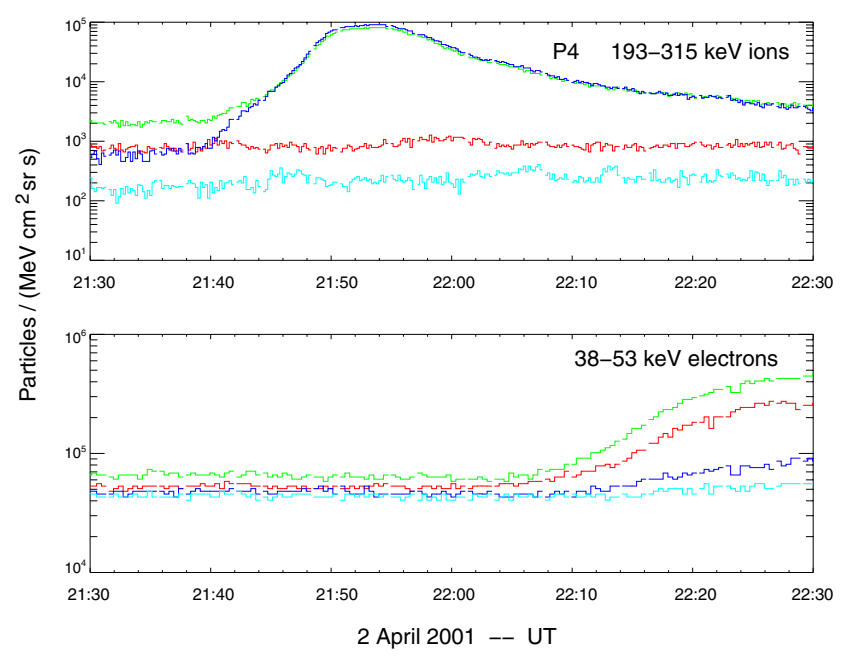

Fig. A.2. As Fig. A.1 except for 2 April 2001.

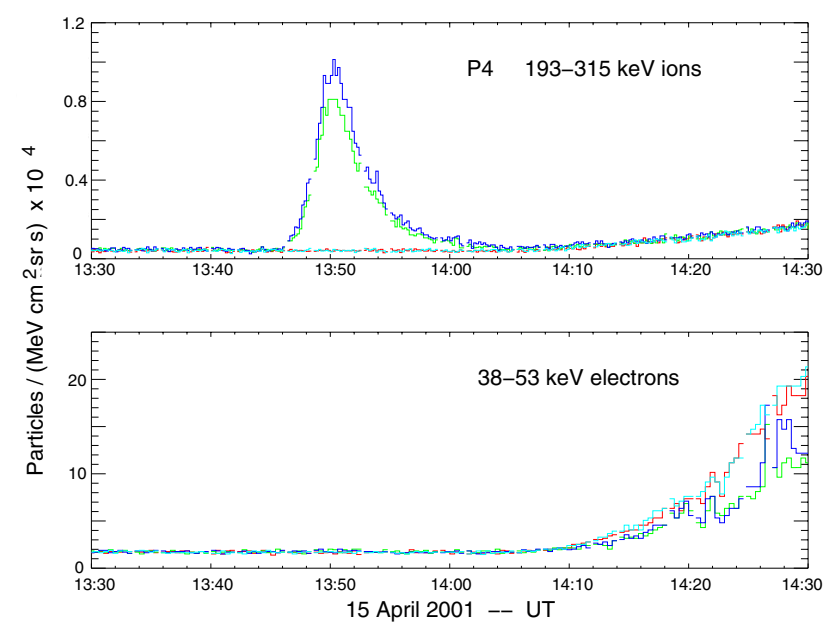

Fig. A.3. As Fig. A.1 except for 15 April 2001.

plotted as red and cyan. The colour code for the electrons is identical. About 20 min later a beam of electrons is detected, which is the normal timing given the longitude of the flare at W33.

The second event we have chosen was GOES class X20, and Fig. A.2 shows the P4 and DE1 channels for the period 21:30-22:30 UT on 2 April, 2001. April 2001 was an active month and the pre-flare background intensity was enhanced over quiet times. Note that the intensities in Fig. A.2 are plotted logarithmically. The $\mathrm{P} 4$ channel responds to the flare X-rays in the blue and green sectors. The electron channel does not repond to the solar X-rays and $\sim 20$ min later the electrons start arriving at ACE as an anisotropic beam. This behaviour is normal for particles associated with a flare near the solar west limb.

Figure A.3 shows the same channels for the time period 13:30-14:30 UT on 15 April 2001. The detector response to the flare is the same as before. Figure A.4 shows the same channels for the period 10:50-11:50 UT on 28 October 2003. The flare was GOES class 17.2 and we have chosen this event partly because there is a very small electron increase aound the onset of the solar X-ray event. However, this is a short-lived beam of solar electrons which must have left the Sun some 15-20 min before the flare onset. We know that the response is not due to X-rays as the peak sector (red) is not a sector that views the Sun, and the sector with the lowest intensity (cyan) is one of the sectors where 


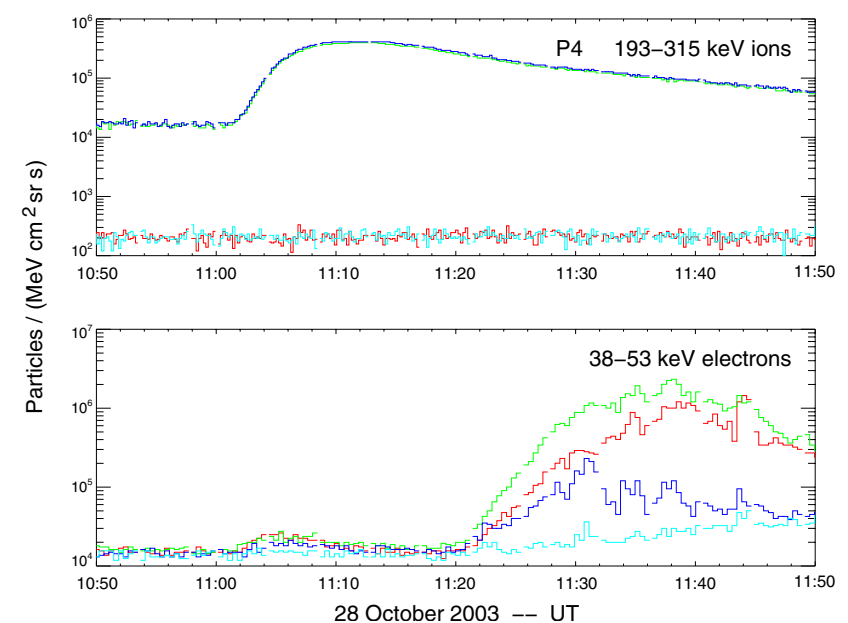

Fig. A.4. As Fig. A.1 except for 28 October, 2003.

the instrument aperture sweeps past the Sun. The 28 October 2003 event has been analysed in depth by Simnett (2005).

The final event we present is that occurring on 6 December, 2006. We have chosen this event as (a) it is a GOES X6.5 flare; and (b) it is the day after the event which is the subject of this paper. Figure A.5 shows the data from 18:30-19:30 UT. The response to this event is essentially identical to that shown in the

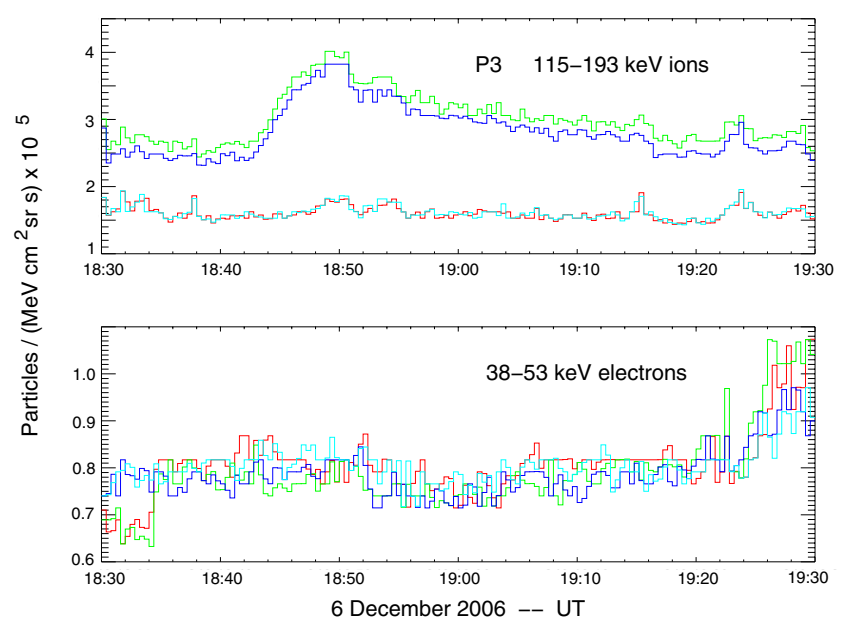

Fig. A.5. As Fig. A.1 except for 2 April 2001, and the upper panel shows the P3 channel of LEMS30, designed to detect ions from 115-193 keV.

previous events, with the two sunward sectors of LEMS30 showing an increase above the (enhanced) background but with no increase in either the non-sunward sectors or the electrons.

The conclusion we draw from this analysis is that the deflected electron channels in the EPAM instrument are not contaminated by solar X-rays. 